\title{
Valutare è ricerca educativa
}

\section{Achille Maria Notti - Rosa Vegliante}

Università degli Studi di Salerno, Dipartimento di Scienze Umane, Filosofiche e della Formazione (DISUFF)

doi: 10.7358/ecps-2014-009-nott

a.m.notti@unisa.it

rosavegliante@gmail.com

\section{EVALUATION: A KIND OF EDUCATIONAL RESEARCH}

\begin{abstract}
Educational assessment, meant as the mere act or effect of the interpretation of student learning outcomes, has greatly changed. The traditional task of assessment is supported by a series of operations setting and interpreting educational acts which affects not only the results, but also the processes and the context in which the actors involved operate in the educational institution at different levels (micro-meso and macro). This kind of evaluation can be considered educational research aimed at gaining a knowledge and understanding of educational events in order to identify problematic nodes with a view to taking action to change and to improve outcomes. Consequently, better results are directly proportional to quality improvement processes. A quality process is characterized and supported by multiple educationallevaluative interventions, which can rationalize decision-making, determine the tasks to be performed, make a change through a planned and contextualized project. Educational outcome measurement is meaningful only if considered from a systemic point of view so as to develop educational research and experiment new teaching strategies.
\end{abstract}

Keywords: Educational research, Evaluation, Experimentation, Quality, Teaching innovation.

Anche in Italia si sta profilando una certa guerra metodologica, spesso più teorica che pratica, tra i fautori di un approccio definibile genericamente sperimentalista e quelli di un approccio che si può dire interpretativista, tra coloro che intendono intervenire per migliorare e quelli che intendono solo descrivere per capire. Per conoscere l'interazione tra un sistema e le relazioni 
che in esso si sviluppano, tra i processi attuati e i risultati attesi, tra il singolo e gli eventi, bisogna affidarsi alla sperimentazione. Fare ricerca significa comprendere per intervenire con l'intenzione di migliorare. Si ricorre alla ricerca per accrescere la conoscenza sui principali nodi problematici di situazioni complesse e di non facile soluzione, al fine di superare quel dualismo tra teoria e prassi, tra il sapere e il saper fare. In questa accezione, la valutazione è chiamata a riconoscere la complessità-processualità dei fatti educativi e, per essere veramente tale, deve non solo confondersi con la ricerca ma divenire strumento di problematizzazione e di indagine. Inizialmente, nell'ambito delle scienze umane, la valutazione è stata considerata una pratica marginale, una forma di ricerca minore poco significativa dal punto di vista dell'elaborazione teorica.

Nel tempo, la produzione scientifica ha evidenziato come numerosi contributi abbiano rivalutato la ricerca "valutativa» rispetto ad altri tipi di ricerca. Tra i vantaggi è stato apprezzato il fatto che si trattasse di un'analisi sul campo, di una fedele riproduzione della realtà, in quanto finalizzata a descrivere ciò che effettivamente accade in un determinato contesto. In generale, i valutatori si avvalgono di una gamma di approcci, derivanti da ambiti disciplinari specifici, senza tralasciare il campo operativo della valutazione che ha in sé questioni peculiari, poco ignorabili (Bezzi, 2007). Non a caso, Palumbo (2001) delinea le cause dell'avanzamento della pratica della valutazione, non più ancilla delle discipline accademiche e degli ambiti professionali, in cui ha avuto origine, ma dotata di una propria autonomia. Tra i fattori di diffusione, la macro-causa si individua nell'avanzamento del carattere democratico e rappresentativo delle istituzioni, che ha contribuito alla delegittimazione delle basi tradizionali dei poteri pubblici. Si ha di fronte un cittadino che chiede allo Stato non solo di giudicare la qualità dei servizi erogati, ma di conoscere l'attuazione dei programmi pubblici e i loro relativi effetti, insomma una domanda di trasparenza e di controllo che è occasione di un miglioramento continuo. Il tema della valutazione, connesso al concetto di qualità e alle sue più disparate accezioni (qualità come rispetto della specificità, come soddisfazione del cliente, qualità percepita, attesa), invade ogni settore, dal sistema aziendalistico-economico alla pubblica amministrazione, dal campo sociologico al contesto formativo/educativo.

Le prime ricerche sulla valutazione educativa prendevano in esame semplicemente l'atto e l'effetto dell'interpretare i risultati dell'apprendimento degli studenti. Nel corso del tempo il suo campo di indagine si è esteso, così che la funzione accertativa del profitto degli studenti è diventata una delle tante funzioni a cui la valutazione deve assolvere. Essa non riguarda solo gli esiti, i prodotti ma si interessa dei processi, del sistema a livello locale (tracciando una panoramica del singolo istituto), a livello meso e macro. La 
valutazione diviene un processo di ricerca interdisciplinare o meglio transdisciplinare (Scriven); è una logica, un modo di pensare che attraversa ogni ambito, se ne avvale e vi fornisce quel quid in più che è il modo di giudicare. Essa è un collante tra fini e mezzi, in quanto non si può ricorrere a strumenti senza avere individuato, preliminarmente, il fine ultimo verso cui indirizzare le azioni educative/formative, né si può raggiungere un determinato risultato senza l'ausilio di mezzi validi ed attendibili.

In una società complessa, dinamica, in continua trasformazione, il sistema scolastico risente dei condizionamenti provenienti dall'esterno che influenzano e modificano la multidimensionalità dei fattori, determinanti ai fini dei risultati del processo educativo. Il passaggio dai saperi cristallizzati e standardizzati, di stampo comportamentista/cognitivista, alla mobilitazione e integrazione degli stessi e delle abilità in contesti situati, diviene espressione di quel particolare costrutto, ascrivibile al concetto di competenza, che connota la contemporaneità e che ha rivoluzionato il modus operandi delle istituzioni deputate alla formazione.

La competenza, di matrice economica e di derivazione europea, viene fagocitata dalle politiche nazionali che regolamentano l'ordinamento scolastico e, con essa, si determina una nuova prospettiva di riferimento all'interno della quale l'innovazione e le nuove proposte educative fungono da oggetto e risultato della ricerca psico-pedagogica e didattica.

Le procedure e le metodologie si diversificano in molteplici e diverse strutture teorico-pratiche che, definendosi in forme specifiche di tecnologia avanzata, concorrono ad acquisire una nuova consapevolezza: non esiste una unicità delle logiche e dei saperi (come, ad esempio, i modelli formativi), quali determinanti esclusivi al cambiamento, bensì una molteplicità di modelli e di strutture teorico-pratiche. E di queste, poi, vanno considerate le intersezioni, gli intrecci che ne determinano l'autentico valore aggiunto (Frabboni \& Pinto Minerva, 1994).

In una cornice dominata da un paradigma di impianto socio-costruttivista, che ridefinisce le pratiche d'insegnamento/apprendimento e dà ampio spazio ad una forma di valutazione alternativa, volta a verificare non solo ciò che uno studente sa ma ciò che "sa fare con ciò che sa» (Wiggins, 1998), trova conferma la teoria vichiana dei corsi e ricorsi storici poiché si riaccende una disputa, iniziata 50 anni orsono, tra i fautori e i detrattori delle prove strutturate.

Da un lato, i sostenitori delle prove strutturate/oggettive, basate su modalità di risposte predefinite, rivendicano l'unicità di tali strumenti, validi e affidabili, nel rilevare non solo conoscenze o abilità decontestualizzate, ma situazioni complesse che richiedono specifiche competenze così come previsto dalle finalità del percorso formativo. In tale prospettiva, ci si avvale delle 
prove strutturate per quantificare il livello di competenza raggiunto dal soggetto, in grado di orchestrare tutte le risorse interne se posto nella condizione di risolvere compiti reali o quanto meno vicini alla realtà. Questi strumenti, la cui valutazione viene sottratta alle caratteristiche soggettive di chi corregge, non annullano comunque, e come molti pensano erroneamente, «'insostituibile momento della scelta soggettiva dei saperi da sottoporre a controllo. Più semplicemente e correttamente di altri lo rendono esplicito attraverso i quesiti e la determinazione delle risposte considerate esatte»(Domenici, 2003, p. 64). Dall'altro lato, gli oppositori delle medesime prove le giudicano inappropriate ai fini di una valutazione di stampo costruttivista, «autentica», tesa a misurare il possesso «di strutture di conoscenza flessibili, la capacità di riorganizzare le loro conoscenze, la capacità di essenzializzare la massa di conoscenze per ridurre il peso cognitivo nel loro uso, la competenza metacognitiva per sapere quando, come e perché è utile applicare determinate strategie» (Comoglio, 2007, p. 4).

I detrattori rivendicano l'impossibilità delle sole prove strutturare di "cogliere alcuni degli elementi essenziali dell'educazione del XXI secolo, come la capacità di problem solving, il pensiero critico, l'intraprendenza e il senso di cittadinanza» (Harlen \& Gardner, 2010) e ravvisano il ricorso ad approcci eclettici poiché l'utilizzo esclusivo di strumenti, così costruiti, ostacola la possibilità di fornire risposte alternative, non preventivamente considerate; non favorisce il pensiero creativo e in più non tiene conto dei processi ma si focalizza sui risultati. Tale dibattito è l'incipit di un discorso allargato che pone il suo focus sulla valutazione educativa e sul suo essere una ricerca educativa.

La valutazione, come pratica sociale, mira ad attribuire senso e valore a fenomeni, situazioni e realtà con le quali entra in contatto. Il fare valutazione induce a riflettere in azione, è una "pratica riflessiva" sull'azione formativa finalizzata a migliorare la qualità del servizio erogato da un'istituzione. Secondo la riflessione epistemologica, condotta da Scriven (2000), la valutazione educativa è intesa come «quel processo con cui si determina il valore, il merito o la significatività di qualche entità; le valutazioni sono il risultato di tale processo. La valutazione può essere esterna o interna, o un insieme di ambedue; quantitativa o qualitativa, o un insieme di ambedue» (p. 39). La valutazione, in particolare, quella formativa, diviene specchio di una specifica realtà, osservata e analizzata secondo diverse prospettive, in grado di evidenziare i punti di forza e di maggiore criticità per intervenire e decidere se modificare, rettificare o sospendere le azioni in atto. Può essere paragonata ad un vero e proprio processo metacognitivo, in quanto consente di prendere coscienza, in maniera consapevole, di ciò che avviene nella situazione fattuale. Non si può pensare di attuare un percorso formativo, separato dal 
processo valutativo, che in una fase iniziale (ex ante) fotografa la situazione di partenza, in una fase intermedia (in itinere) monitora il percorso rispetto a quanto progettato e, in fase conclusiva (ex post) mira a stilare un bilancio dell'esperienza educativa realizzata. Conveniamo con Galliani (2009) che la valutazione educativa è finalizzata ad emettere giudizi sulle azioni formative, progettate a questo scopo, e indirizzate ad orientare e a sviluppare apprendimenti negli allievi, con ricadute sui sistemi formativi, economici e sociali. Essa è fondata sull'uso di metodi e strumenti propri della ricerca empirica e sperimentale.

Le valutazioni esterne condotte da organismi internazionali (IEA-PIRLS, OCSE-PISA), negli ultimi decenni hanno registrato una carenza, da parte dei nostri studenti, in prove mirate a testare la competenza nella lettura e la competenza nell'ambito scientifico-matematico rispetto agli esiti conseguiti dagli studenti appartenenti ai Paesi del Nord Europa. A livello nazionale, si allarga la forbice tra le scuole di eccellenza e le scuole «scadenti» e, anziché cercare di comprendere il perché si è giunti a tali risultati, attuando un'analisi sistemica che prendesse in considerazione sia la pluralità di variabili, che incidono e condizionano il processo formativo (realizzato nelle singole realtà scolastiche), sia l'affermarsi di modelli di culture sociali, che influiscono sulle scelte formative, si è proceduto con rattoppi senza alcuna visione prospettica. Tale è stata la reazione delle scuole che hanno considerato il modello valutativo «esterno», basato sulla misurazione degli esiti, lo strumento per scaricare su di esse le difficoltà del sistema.

Per innescare un processo migliorativo non basta attenersi semplicemente a una valutazione proveniente dall'esterno basata, esclusivamente, sulla comparazione di dati quantitativi e avulsa da contesti di autoanalisi di istituto. A partire dalla misura degli esiti formativi rilevati, bisogna attuare una valutazione di natura sistemica che sia in grado di conferire senso e significato alla totalità, a partire dall'analisi delle sue parti, e che si focalizzi non solo sugli esiti, ricavati da strumenti standardizzati mediante prove oggettive (volte a quantificare il possesso di una determinata conoscenza/competenza in riferimento ad una data prestazione) ma soprattutto: sui processi, sul binomio mezzi-fini, sulla qualità dell'insegnamento/apprendimento, sulle risorse umane, sui materiali a disposizione, con l'obiettivo di sviluppare la ricerca e sperimentare nuove strategie di insegnamento. Valutare significa leggere in maniera significativa l'esperienza in atto, è un processo di interpretazione, comprensione e metacognizione, in grado di acquisire e fornire dati informativi e conoscitivi. Un servizio orientato alla qualità dell'istruzione non si limita alla sola rilevazione di variabili dipendenti, ma deve cogliere le trasformazioni del quadro formativo per consentire di operare rettifiche tempestive nell'organizzazione scolastica e negli interventi didattici. 
Gli esiti delle ricerche nazionali e internazionali vanno inseriti in uno scenario allargato che ci permette una contestualizzazione non improvvisata dei problemi emergenti. D'altronde, il senso dei risultati delle azioni educative viene conferito sulla base di un processo successivo che deve riflettere sui risultati conseguiti per poi ripartire dai medesimi risultati. Nella processualità delle azioni educative, influenzata da variabili individuali, didattiche, organizzative, è rinvenibile il senso dei risultati conseguiti, posti in stretta correlazione con il processo sistematico di autovalutazione di istituto. Entro tale logica sistemica, l'autovalutazione mira a comprendere, in maniera olistica e situata, i molteplici fattori che incidono sull'intero processo educativo-formativo sia per ciò che attiene la didattica e le strategie d'insegnamento, messe in campo dai docenti, sia per ciò che riguarda l'impianto organizzativo e gestionale delle risorse materiali, economiche, umane a disposizione. In un frame integrato è possibile ovviare ad azioni, semplicistiche e riduzionistiche, connesse all'esclusivismo dei soli esiti rilevati, poiché la sola misurazione dei risultati non fornisce informazioni utili alla correzione e alla riprogettazione dei processi attuati. Il miglioramento dei risultati è direttamente proporzionale al miglioramento della qualità dei processi. Un processo è di qualità nel momento in cui è accompagnato e sostenuto da più interventi educativi/valutativi mirati a razionalizzare le scelte effettuate, a individuare le attività da svolgere, attraverso una progettualità, pensata e contestualizza, tesa alla realizzazione degli obiettivi da perseguire. Alla valutazione esterna, pratica condotta da organismi deputati a promuovere la crescita e il miglioramento delle istituzioni autonome, va necessariamente affiancato un processo di riflessione, proveniente dall'interno, che vede il coinvolgimento attivo dell'unità scolastica, con l'obiettivo di promuovere gli incrementi degli aspetti qualitativamente più efficaci ed apprezzabili del sistema scolastico e rendere recessivi, invece, quegli elementi che risultano scarsamente efficaci (Notti, 2003). Dunque, sulla scia di Domenici (2000), va ribadito che la valutazione interna alla singola scuola consente di cogliere il legame tra le prestazioni raggiunte dagli alunni, appartenenti a quella specifica realtà scolastica, e le prestazioni standard, definite a livello nazionale o internazionale, così da accertarne la perequazione o lo scarto, in termini di vantaggio/svantaggio formativo. I processi di autovalutazione consentono di colmare quel gap tra la scuola, istituzione dotata di una propria autonomia organizzativa e gestionale, e ciò che avviene al suo interno, in rimando alle varie progettualità didattico-formative realizzate.

In tal senso, l'oggetto della valutazione interna è l'efficacia degli obiettivi definiti dall'organizzazione e l'efficienza nell'utilizzazione delle risorse; il fine della valutazione esterna, invece, è quello di valutare in che modo il processo organizzativo ha raggiunto gli obiettivi definiti a livello di sistema (Allulli, 2000). 
La valutazione, pensata in chiave costruttivista o di «quarta generazione» (Guba \& Lincoln, 2007), la si può paragonare ad un processo aperto in continua tras-formazione in cui il valutatore, agendo insieme agli stakeholders nel loro ambiente naturale, favorisce l'emergere dei problemi e scopre risultati che non potevano essere previsti in anticipo. A partire dall'individuazione e dall'analisi delle variabili, da cui dipendono i risultati del processo educativo, la valutazione diviene l'unico mezzo in grado di innescare processi autoriflessivi e responsabili che tengano conto degli obiettivi ipotizzati e delle finalità educative (così come indicate dalla normativa), in vista di un miglioramento continuo delle pratiche adottate. Ciononostante, è necessario avvalersi di più strumenti di analisi e di valutazione, indirizzati a rilevare informazioni pertinenti su una pluralità di fattori, che sono determinanti ai fini della qualità dell'offerta formativa e che possono essere sintetizzati nei seguenti interrogativi:

- qual è la finalità del processo formativo (apprendimento di conoscenze, competenze);

- come viene favorito l'apprendimento (processo formativo, qualità della didattica, relazioni ed inclusione);

- in quale condizione si realizza il processo educativo (autonomia e gestione scolastica).

Le risposte a tali interrogativi, strettamente dipendenti e conseguenziali, permettono di far luce sugli elementi che costituiscono e qualificano il microsistema educativo.

Il prodotto formativo degli allievi funge da criterio di riferimento per valutare la produttività del servizio scolastico. Sul piano didattico, cruciale importanza è riconosciuta al processo di traduzione dei traguardi formativi in conoscenze e abilità cognitive complesse, spendibili in situazioni che non rispecchiano gli originali contesti di apprendimento. Nell'attuale enfasi, la principale finalità del processo formativo consiste nell'apprendimento di competenze. Questo viene garantito nel momento in cui gli interventi educativi, pensati e agiti, all'interno di una progettualità responsabile, flessibile e integrata nei diversi contesti, siano in grado di coniugare i bisogni dell'utenza con le esigenze dell'istruzione, declinati in termini di traguardi formativi. La grande sfida della scuola contemporanea consiste nel coniugare posizioni costruttiviste, socio-costruttiviste con posizioni cognitiviste e neocomportamentiste. Da un lato, i sostenitori di un apprendimento in situazione, fondato su compiti complessi; dall'altro i fautori di un apprendimento graduale, step by step, primo passo necessario per acquisire saperi più complessi. La dialettica, dal semplice al complesso, pervade il contesto di apprendimento, il cui scopo risiede nel rintracciare sia elementi di continuità con la realtà esterna, che dia agli studenti la possibilità di confrontarsi con situazioni autenti- 
che, sia elementi di discontinuità rispetto ad esperienze extra-scolastiche, «in relazione alla loro funzione preparatoria e vicariante rispetto alla vita, che esige gradualità, attenzione ai ritmi di apprendimento individuali» (Maccario, 2012, p. 113). In questo modo, gli studenti acquisiscono solide basi per affrontare esperienze formative future. Va da sé che la qualità del profitto scolastico, o meglio, dell'apprendimento, non dipende esclusivamente dall'ottimizzazione degli interventi realizzati. Il processo formativo, messo in atto, è fondamentale per la qualità dell'apprendimento ma non è esaustivo, in quanto l'incidenza di fattori ambientali, socio-culturali, motivazionali, relazionali, etc., gioca un ruolo importante sulla qualità dell'apprendimento (Notti, 2010). In esso, infatti, convergono la competenza degli operatori, un'attenta progettazione, una coerente organizzazione delle attività, accompagnati dal processo valutativo che oltre ad essere credibile deve anche apparire tale.

Una scuola di qualità offre servizi in grado di promuovere l'apprendimento e la crescita culturale e professione degli attori coinvolti. Spesso, si afferma che il prodotto della scuola è la cultura e, così, si rischia di confondere il fine con il mezzo, di scambiare il prodotto con quanto è necessario per produrlo. Del resto, quando si fanno coincidere i risultati degli studenti con il prodotto della scuola, si tende a sottovalutare l'unicità e la complessità dell'azione educativa. Entro tali posizioni, si può cogliere la meta-finalità del processo formativo che consiste nella costruzione strutturata ma flessibile di opportunità che stimolano e motivano l'apprendimento e il percorso di formazione degli alunni (Notti, 2010). La valutazione ci permette di osservare e controllare il reale profitto che gli allievi traggono dal servizio offerto, con l'obiettivo di intervenire per costruire e realizzare le opportunità formative ideate.

L'accessibilità ai contenuti del sapere è garantita dalla pratica della mediazione didattica, nel momento in cui il docente riesce a coniugare gli oggetti culturali con i contenuti scolastici e a tradurli in termini di obiettivi da raggiungere. Il processo attuato non deve essere una replica di un'esperienza precedente, non va inteso come un insieme di procedure prestabilite, ma è il modo migliore per affrontare e risolvere problemi, puntando sullo sviluppo innovativo delle attività formative (Lichtner, 1999). La valutazione del processo formativo deve mirare a:

- stabilire le soglie entro le quali individuare d'aver raggiunto gli obiettivi programmati;

- stabilire le azioni da compiere per ottenere i risultati richiesti;

- prevedere azioni di recupero, nel caso siano necessarie;

- provvedere alle risorse in termini di mezzi, professionalità e tempi;

- rispondere alle esigenze dei destinatari degli interventi.

Va ribadito che, in questo caso, la valutazione non giudica ma supporta e accompagna il percorso formativo, divenendo occasione di riflessione, di 
condivisone, di confronto da parte dei soggetti coinvolti nello stesso processo.

La buona riuscita del servizio erogato dalla scuola è garantire a tutti e a ciascuno effettive opportunità di apprendimento, un compito non facile, data la molteplicità dei fattori implicati.

Uno tra i tanti è rappresentato dalla didattica con le innumerevoli variabili di cui si compone «non solo di ordine cognitivo, perché un ruolo determinate è svolto da fattori non cognitivi, fra cui rientrano quelli motivazionali o legati alla percezione delle proprie competenze» (Gattico \& Mantovani, 1998 , p. 96). Nel processo valutativo non si tiene conto solo delle performance degli studenti, sono coinvolti anche gli insegnanti con il loro operato, attraverso un modello partecipativo e collaborativo, volto al raggiungimento di una didattica di qualità, in cui gli attori in gioco sono chiamati a partecipare, in maniera attiva, secondo un modello di tipo bottom-up. Monitorare il processo di insegnamento/apprendimento nelle varie dimensioni (relazionale, organizzativa, metodologica) consente di controllare in fieri le ricadute a livello sociale rispetto alle azioni e alle strategie didattiche messe in campo, ai fini del raggiungimento dei risultati auspicati. Tutto ciò, naturalmente, va pensato all'interno di un habitat "favorevole» che la singola scuola, dotata di autonomia, deve poter creare. L'autonomia va intesa come un paradigma politico-sociale-pedagogico che riguarda sia il management scolastico sia l'organizzazione della didattica e dei percorsi formativi. Il giudizio negativo della scuola non è scisso dall'impegno e dalla responsabilità dei protagonisti coinvolti, la cattiva qualità del servizio coinvolge tutti. L'autonomia, per gli istituti scolastici, è sinonimo di emancipazione rispetto ad una politica accentratrice, con essa la scuola vive l'autogoverno in termini economici, progettuali, di ricerca e di sperimentazione dei processi rispetto al servizio progettato ed effettivamente prestato. Come sistema complesso-autonomo, si specializza nella gestione, nell'organizzazione e nella realizzazione di servizi sulla base dei bisogni dell'utenza. In tal modo, abbandona il modello centralistico e burocratico, e si rende, quindi, responsabile nell'autogestire, al meglio, le risorse umane, economiche e materiali, facendo giocoforza sul coinvolgimento attivo e partecipativo dei vari operatori scolastici. Le istituzioni scolastiche costruiscono la loro mission formativa adeguando le Indicazioni Nazionali con le caratteristiche contestuali, nelle quali la scuola opera. Ovviamente, gli standard di qualità del servizio offerto sono stabilite in linea con le finalità formative da raggiungere. L'autonomia delle scuole è indirizzata a favorire lo sviluppo e la crescita dei soggetti, a partire dagli allievi e dalle professionalità interne, considerando le diverse soggettività, elementi codeterminanti ai fini della qualità e dell'efficienza formativa.

Quindi, pur considerando il ruolo assunto dalle variabili che determinano la qualità della didattica (livello micro), va sottolineata anche la rilevanza 
di quelle variabili che si collocano a livello meso e macro, quali l'organizzazione dei processi interni, la qualità del management, i criteri e le modalità di controllo del rendimento scolastico, le relazioni e i rapporti con la comunità scolastica, i rapporti con il mondo produttivo, le risorse umane (professionali) e finanziare (Gattico \& Mantovani, 1998).

Da tale discorso scaturisce che le scelte dettate dalle politiche scolastiche delineano:

- le linee di sviluppo del sistema;

- l'organizzazione delle scuole;

- i profili professionali degli insegnanti;

- la sperimentazione didattica.

Le linee di sviluppo del sistema educativo mirano ad innalzare i livelli complessivi di qualità dell'offerta formativa, sulla base di un approccio integrato e combinato tra l'orientamento top-down, proveniente dai risultati desunti dall'accountability esterna, e quello bottom-up, tipico dell'improvement interno. I compiti istituzionali svolti dalle scuole, presenti sul territorio, vengono perseguiti avvalendosi di strategie, soluzioni, procedure, relazioni, comportamenti che vanno a costituire il modello organizzativo che la scuola si dà per conseguire i propri fini. «Senza organizzazione, nessuna situazione formativa verrebbe ad esistere, nessuno spazio verrebbe aperto, come luogo definito" (Lichtner, 1999, p. 215). L’organizzazione scolastica è la cornice entro cui si sviluppa il processo formativo; in essa acquistano senso e significato l'insieme delle azioni e degli interventi educativi da realizzare, in corrispondenza con i bisogni individuali e collettivi. L'organizzazione è tesa alla valorizzazione delle professionalità e delle esigenze degli allievi e della committenza. In questa accezione, bisogna creare una cultura dell'organizzazione, intesa come processo complessivo che preveda una visione d'insieme delle procedure, delle interazioni, delle decisioni che si pongono ad una istituzione scolastica, mettendo da parte il modo tradizionale di considerare l'organizzazione come un insieme di azioni codificate e segmentate. Per rispondere efficacemente ed efficientemente agli studenti e alle istanze territoriali c'è bisogno di una organizzazione interna, anch'essa efficace ed efficiente e, per questo, di un'articolazione dei livelli di responsabilizzazione e di decisionalità adeguati ai problemi da affrontare. Linterazione tecnico/ personale docente, la collaborazione tra i docenti, una sburocratizzazione dei comportamenti, il decentramento delle responsabilità, la scansione dei tempi, la gestione e l'attuazione dei processi formativi, sono solo alcune variabili che necessitano di un sistema organizzativo. L'organizzazione delle scuole deve possedere tre requisiti: la razionalità, la formalizzazione e il conseguimento degli scopi. Essa si definisce razionale quando si fonda su un modo di operare pianificato e programmato che prevede di ottimizzare le risorse e le azioni, non in maniera 
casuale o accidentale, ma secondo quanto stabilito in precedenza. Dalla razionalità scaturisce il secondo criterio: la formalizzazione. La formalità dell'organizzazione scolastica si manifesta attraverso procedure esplicite e norme impersonali, garantite dalle varie figure di coordinamento. Il terzo requisito, il conseguimento degli scopi, rinvia all'obiettivo prioritario dell'organizzazione, ossia al successo formativo (Crispiani, 2010). Monitorare il modello organizzativo, adottato dalla scuola, significa descriverne i livelli di efficienza, la sua capacità di gestire ed ottimizzare il servizio a partire da situazioni date; significa rilevare la qualità della spesa, l'impiego delle risorse umane e materiali, la reattività nel rispondere a situazioni impreviste, ma anche descrivere l'apporto dei soggetti a cui questo compito è demandato. L'organizzazione scolastica è tutto ciò che regola la pianificazione della vita scolastica intesa nella sua globalità. Pertanto il servizio educativo richiede l'organizzazione di un sistema formativo integrato, che vede l'istaurarsi di relazioni di reciprocità tra la scuola, il territorio e gli enti in esso presenti.

Il framework delle competenze di base, messo a punto nel 2006 dal Parlamento Europeo e dal Consiglio d'Europa (2006/962/E), è parte integrante di quel quadro normativo nazionale che influenza notevolmente il profilo professionale degli insegnanti. Come sostiene Bottani (1994), «i docenti non nascono a caso, non sono il risultato di un'evoluzione spontanea, di una vocazione professionale, di una maturazione di doti e di attitudini naturali; essi sono piuttosto il prodotto di un'operazione molto elaborata e complessa, messa a punto quando si è capito che l'istituzione formale scolastica costituisce la modalità più efficace per agire sugli uomini» (p. 24). Le vertiginose trasformazioni sociali, economiche, politiche incidono sul quadro formativo dei docenti. L'insegnante risente dei cambiamenti provenienti dall'esterno e, più di ogni altra professione, si attiva nel rispondere alle innovazioni tecnologiche e didattiche, investendo nella formazione continua e nell'aggiornamento personale. L'esigenza di aggiornamento costante si lega alla necessità di formare un cittadino attivo che sia capace di imparare ad imparare, secondo una logica di longlife learning, che rende obsoleta la separazione tra educazione formale e non formale. Per colmare i bisogni di aggiornamento, va intrapresa la via della formazione continua. Questo processo impegna ogni persona nello sviluppo, arricchimento ed allenamento del proprio intelletto. L'insegnate matura l'esigenza di aggiornare le proprie competenze sia dal punto di vista disciplinare sia rispetto all'ambito metodologico-didattico, in un'attività che si ripete periodicamente e che consente il confronto tra le esperienze. Per mediare tra la competenza (il nuovo baricentro del curricolo) e il processo formativo, indossa le vesti di un professionista riflessivo (Schön, 1993) che, durante l'azione d'insegnamento, è in grado di applicare la tecnica avvalendosi di un approccio poietico. Riflettere in azione e passare dalla 
«logica dell'insegnamento» alla «logica dell'apprendimento» sono le grandi sfide che il docente contemporaneo si propone di affrontare. In una prospettiva di derivazione socio-costruttivista, l'insegnate diviene colui che sposta l'asse dal dentro/scuola al fuori/scuola, crea dei link tra il sapere formale e il sapere informale ed è chiamato a svolgere una funzione di facilitatore della negoziazione e della comunicazione tra gli studenti. L'apertura con la realtà circostante non è da considerarsi una minaccia, così da appiattire il ruolo a cui è deputata l'educazione formale, tutt'altro, l'insegnamento assume la conoscenza come evento complesso, globale, situato, multidimensionale, non tende a frazionare il sapere in componenti elementari per renderlo più accessibile, ma dà vita ad una relazione ricorsiva tra esperienza e conoscenza stessa, tra teoria e pratica. Il docente, oltre ad essere dotato di specifiche competenze disciplinari, diviene un "progettista» della formazione in grado di pianificare e organizzare il processo di insegnamento/apprendimento, ponendovi al centro le esigenze dell'allievo. Le attività formative vengono pianificate, organizzate, in rapporto ai criteri generali, di carattere nazionale ed internazionale, e ai criteri assunti a livello locale e contestuale. Per progettare un percorso efficace ed efficiente, il docente-progettista deve focalizzarsi principalmente sul cosa fornire agli allievi e sul come fornirlo. Il cosa rinvia alla qualità della competenza professionale, alla capacità di riscuotere fiducia ed autorevolezza, di essere considerati affidabili. Il come fa riferimento al tipo di relazioni che si istaurano, alla capacità di stimolare interesse e di motivare gli allievi (Notti, 2010).

Nelle Indicazioni Nazionali 2012 si legge che «la professionalità docente si arricchisce attraverso il lavoro collaborativo, la formazione continua in servizio, la riflessione sulla pratica didattica, il rapporto adulto con i saperi e la cultura. La costruzione di una comunità professionale ricca di relazioni, orientata all'innovazione e alla condivisione di conoscenze, è stimolata dalla funzione di leadership educativa della dirigenza e dalla presenza di forme di coordinamento pedagogico». Al di là dei dettami normativi, un insegnamento realmente efficace prevede un lavoro sinergico e una corresponsabilità condivisa da tutta l'équipe.

Dunque, per realizzare una didattica di qualità è necessario puntare e investire sulla ricerca/sperimentazione didattica, finalizzata all'adozione di pratiche didattiche innovative e flessibili, di strategie che si adeguino alle esigenze degli allievi e alle caratteristiche dei contesti socio-culturali. La sperimentazione, nell'ambito della didattica, contrasta con le condizioni già date, caratterizzate da «materiali didattici prefabbricati, monoprocedurali, iperformalizzati [...]. Lo stile sperimentale è una sorta di termostato didattico in grado di mantenere in equilibrio dialettico due ineludibili postulati pedagogici. Da una parte, il rispetto delle motivazioni, esperienze dell'utenza 
scolastica; dall'altra parte, una linea formativa antidogmatica e antiautoritaria, possibile a partire da una scuola rispettosa (e quindi attenta) della pluralità dei punti di vista metodologici» (D’Amore \& Frabboni, 2005, p. 20). La ricerca educativa getta le basi su una cultura dell'innovazione che si propone di migliorare le azioni educative in vista delle prestazioni attese. Muoversi in questa direzione significa trasformare la sporadicità in sistematicità, considerare la sperimentazione come forma di anticipazione del futuro o come forma di applicazione di un orientamento normativo non ancora chiaro e definito. La sperimentazione didattica è un processo collettivo e sociale, è un'ipotesi nuova rispetto all'esistente e su di essa viene auspicata la speranza di innalzare il valore della singola scuola.

Per superare la frammentarietà degli interventi e poter gestire la complessità, che invade il sistema educativo, l'impianto organizzativo/strutturale e il funzionamento di ogni singola scuola viene garantito dall'attuazione di un progetto educativo a medio/lungo termine. Non si progetta per un tempo indefinito, le azioni designate all'interno del progetto formativo sono connesse ad un bisogno educativo che muta nel tempo e che, per essere soddisfatto, richiede un'interazione continua tra il docente e l'allievo, tra l'istituto scolastico e il contesto e tra quest'ultimo e il sistema formativo. Il progetto non è semplicemente un'ideologia ma una strategia operativa, in quanto è sorretto da una struttura metodologica rigorosa. Al suo interno, deve poter coniugare l'intenzione espressa, qui ed ora, in maniera vaga e precisa, da realizzare, e il particolare organizzato, ossia la previsione di ciò che si intende fare: il disegno, il modello, lo schema (Ardoino, 1977).

Nella scuola dell'autonomia, la progettazione, caratterizzata dal momento dell'ideazione, della formulazione e della realizzazione concreta, favorisce la costruzione di processi educativi e formativi, mediante attività svolte con risorse, metodologie e tecniche appropriate, accompagnate da un processo valutativo teso a provarne l'efficacia e, in caso di riscontro negativo, predisporre una riformulazione degli interventi. L'articolazione progettuale prevede di chiarire e definire le finalità generali e gli obiettivi da raggiungere, attraverso forme realisticamente compatibili con gli interventi da mettere in campo, che non possono prescindere dalla ricognizione delle risorse disponibili (umane, finanziarie e materiali). A tal proposito, va evidenziato che il passaggio dall'ideazione all'attuazione del progetto educativo è assicurato solo se a monte sussistono due importanti precondizioni: lo sviluppo della sperimentazione e la condivisione del personale scolastico. In qualsiasi contesto in cui si progetta si fa ricerca. L'azione del progettare è il risultato di decisioni assunte sulla base di indagini conoscitive, di valutazioni effettuate e di eventuali previsioni future. Volendo riprendere Cattaneo (2004) sono tre le dimensioni che conferiscono senso all'azione progettuale: 
- il coinvolgimento, personale e professionale, da parte di chi è implicato nell'ideare il progetto;

- la consapevolezza che ogni istituzione costituisce la propria identità (e lascia tracce nella storia);

- la valutazione del progetto, pratica che consente di cogliere le criticità e le potenzialità e mira a ridurre le prime e a innalzare le seconde.

Sperimentare diviene sinonimo di cambiamento. Intervenire per accrescere la qualità del servizio, per riqualificare gli interventi educativi dell'istituto, è la risposta all'innovazione. La progettazione deve essere reale, situata, non può affrontare problemi generali, deve riferirsi alla cultura dominante nel contesto in cui è pensata, richiede l'interconnessione di specifiche competenze organizzative, comunicative, relazionali. Le competenze richiamate e il collegamento tra di esse «rende la progettazione strumento autorevole di qualificazione dei processi formativi, i quali, sollecitano, a seconda dei tempi e delle occasioni, interventi calibrati, su misura dei soggetti e in considerazione di contesti di vita coinvolti» (Orefice, 1997). Quindi, le risorse, i vincoli, le regole, le scelte, le professionalità degli attori implicati, sono parte integrante di un sistema organico costantemente alimentato dalle interazioni tra saperi, competenze e relazioni.

Ogni progetto educativo rompe con la consuetudine, con la ripetibilità delle azioni didattiche e, in quanto tale, ha una sua periodizzazione, ha un inizio e una fine; nasce nel momento in cui si individua un problema reale, un bisogno da colmare che, attraverso opportune strategie, giunge al termine non appena soddisfatto.

\section{RIFERIMENTI BIBLIOGRAFICI}

Allulli, G. (2000). Le misure della qualità. Roma: Seam.

Ardoino, J. (1977). Education et politique. Paris: Gauthier Villars.

Bezzi, C. (2007). Che cos'è la valutazione. Milano: Franco Angeli.

Bottani, N. (1994). Professoressa, addio. Bologna: Il Mulino.

Cattaneo, P., Marotta, E., \& Tartarelli, A. (2004). La progettazione formativa nella scuola. Catania: La Tecnica della Scuola.

Coggi, C., \& Notti, A. M. (a cura di). (2003). Docimologia. Lecce: Pensa.

Comoglio, M. (2007). Valutazione autentica. L'Educatore, 11, Maggio.

Crispiani, P. (a cura di). (2010). Il management nella scuola di qualità. Roma: Armando.

D’Amore, B., \& Frabboni, F. (2005). Didattica generale e didattica disciplinare. Milano: Mondadori. 
Domenici, G. (2000). La valutazione come risorsa. Analisi degli apprendimenti e autovalutazione d'istituto. Napoli: Tecnodid.

Domenici, G. (2003). Manuale della valutazione scolastica. Roma - Bari: Laterza.

Frabboni, F., \& Pinto Minerva, F. (1994). Manuale di pedagogia generale. Roma Bari: Laterza.

Galliani, L. (a cura di). (2009). Web ontology della valutazione educativa. Lecce: Pensa Multimedia.

Gattico, E., \& Mantovani, S. (a cura di). (1998). La ricerca sul campo in educazione. I metodi quantitativi. Milano: Bruno Mondadori.

Guba, E. G., \& Lincoln, Y. S. (2007). La valutazione di quarta generazione. In N. Stame (a cura di), Classici della valutazione. Milano: Franco Angeli.

Harlen, W., \& Gardner, J. (2010). Assessment to support learning. In J. Gardner, W. Harlen, L. Hayward, \& G. Stobart, Developing teacher assessment. Berkshire: Open University Press.

Lichtner, M. (1999). La qualità dell'azione formativa. Milano: Franco Angeli.

Maccario, D. (2012). A scuola di competenze. Verso un nuovo modello didattico. Torino: SEI.

Notti, A. M. (2010). Valutazione e contesto educativo. Lecce: Pensa.

Orefice, P. (a cura di). (1997). Formazione e processo formativo: ipotesi interpretative. Milano: Franco Angeli.

Palumbo, M. D. (2001). Il processo di valutazione. Decidere, programmare, valutare. Milano: Franco Angeli.

Schön, D. A. (1993). Il professionista riflessivo. Per una nuova epistemologia della pratica professionale. Bari: Dedalo.

Scriven, M. (2000). La valutazione: una nuova scienza. In A. Bondioli \& M. Ferrari (a cura di), Manuale di valutazione del contesto educativo: teorie, modelli, studi per la rilevazione della qualità della scuola. Milano: Franco Angeli.

Wiggins, G. P. (1998). Educative assesment: Designing assessment to inform and improve student performance. Roma: Armando.

\section{RiassunTO}

La valutazione educativa, intesa come il semplice atto o effetto dell'interpretare $i$ risultati dell'apprendimento degli studenti, ha subito un profondo ripensamento. Il compito tradizionale della valutazione è affiancato da un complesso processo di regolazione ed interpretazione degli interventi educativi che non riguarda solo gli esiti, i prodotti ma si interessa dei processi, del contesto in cui operano gli attori coinvolti nell'istituzione scolastica a vari livelli (micro-meso e macro). In tal senso, la valutazione è ricerca educativa poiché mira 
alla conoscenza e alla comprensione dei fatti educativi al fine di individuare i nodi problematici per intervenire con l'intenzione di migliorare. Ne consegue che il miglioramento dei risultati è direttamente proporzionale al miglioramento della qualità dei processi. Un processo è di qualità nel momento in cui è accompagnato e sostenuto da più interventi educativi/valutativi, che sono in grado di razionalizzare le scelte effettuate, di individuare le attività da svolgere, di apportare cambiamento attraverso una progettualità pensata e contestualizza. La misura degli esiti formativi rilevati acquista significato solo se interpretata in chiave sistemica con l'obiettivo di sviluppare la ricerca educativa e sperimentare nuove strategie didattiche.

Parole chiave: Innovazione didattica, Qualità, Ricerca educativa, Sperimentazione, Valutazione. 